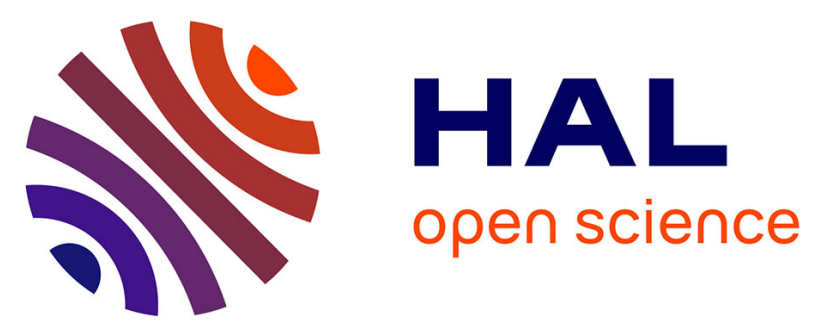

\title{
Steady-state thermal gradient induced by pulsed laser excitation in a ferromagnetic layer Steady-state thermal gradient induced by pulsed laser excitation in a ferromagnetic layer
}

\author{
S Shihab, L. Thevenard, Aristide Lemaître, J.-y Duquesne, C. Gourdon
}

\section{To cite this version:}

S Shihab, L. Thevenard, Aristide Lemaître, J.-y Duquesne, C. Gourdon. Steady-state thermal gradient induced by pulsed laser excitation in a ferromagnetic layer Steady-state thermal gradient induced by pulsed laser excitation in a ferromagnetic layer. Journal of Applied Physics, 2016, 119 (15), pp.153904. 10.1063/1.4947226. hal-01438597

\section{HAL Id: hal-01438597 \\ https://hal.science/hal-01438597}

Submitted on 17 Jan 2017

HAL is a multi-disciplinary open access archive for the deposit and dissemination of scientific research documents, whether they are published or not. The documents may come from teaching and research institutions in France or abroad, or from public or private research centers.
L'archive ouverte pluridisciplinaire HAL, est destinée au dépôt et à la diffusion de documents scientifiques de niveau recherche, publiés ou non, émanant des établissements d'enseignement et de recherche français ou étrangers, des laboratoires publics ou privés. 


\title{
Steady-state thermal gradient induced by pulsed laser excitation in a ferromagnetic layer
}

\author{
S. Shihab, ${ }^{1}$ L. Thevenard, ${ }^{1}$ A. Lemaitre ${ }^{2}$ J.-Y. Duquesne, ${ }^{1}$ and C. Gourdon ${ }^{1}$ \\ ${ }_{1}^{1}$ Institut des NanoSciences de Paris, Sorbonne Universités, UPMC Univ Paris 06, CNRS-UMR 7588, \\ F-75005 Paris, France \\ ${ }^{2}$ Laboratoire de Photonique, de Nanostructures, CNRS, Université Paris-Saclay, route de Nozay, \\ F-91460 Marcoussis, France
}

(Received 19 January 2016; accepted 8 April 2016; published online 20 April 2016)

\begin{abstract}
In all-optical pump-probe experiments on ferromagnetic layers, the determination of the temperature under the pump laser spot is crucial for a quantitative modeling of the magnetization dynamics. We present here a method to quantify this thermal gradient, exemplified on a (Ga, Mn)(As, P) ferromagnetic semiconductor layer on a GaAs substrate. To estimate the local steady-state temperature, we use the coercive field as a thermometer. The probe records the hysteresis cycle spatially across the hot spot, using the magnetic linear birefringence/dichroism of the sample. Our results are analyzed using the heat diffusion equation with two fitting parameters, the thermal conductivity of the layer/ substrate sample and the thermal resistance between the substrate and the thermostat. This opens the way to a quantitative modeling of laser pulse-triggered magnetization dynamics in the presence of transient temperature effects. Published by AIP Publishing. [http://dx.doi.org/10.1063/1.4947226]
\end{abstract}

\section{INTRODUCTION}

Heat dissipation is a critical issue in modern information technology because of the reduced size of the components. In an attempt to overcome this problem, new spintronics or magnonics concepts are developed ${ }^{1,2}$ in order to generate and process information while limiting the heat production. One such possibility relies on the interaction of light with magnetization in order to assist or induce magnetization reversal $^{3,4}$ or to excite spin waves. ${ }^{5-10}$ To investigate the ultrafast all-optical manipulation of magnetization, laser pump-probe experiments are used, ${ }^{11}$ in which the pump laser pulses trigger the magnetization dynamics. However, thermal effects may still be present even under laser excitation. Indeed, the excitation of the magnetization dynamics often relies on a transient thermal variation of the magnetization modulus $^{5}$ or of the magnetic anisotropy constants. ${ }^{12}$ These mechanisms are particularly efficient in the ferromagnetic semiconductors ( $\mathrm{Ga}, \mathrm{Mn}) \mathrm{As}$ and $(\mathrm{Ga}, \mathrm{Mn})(\mathrm{As}, \mathrm{P})$, where the anisotropy constants $K_{i}$ strongly depend on the temperature. $^{13}$ The variation of these parameters with the pump $\delta K_{i} / K_{i}$ not only depends on the transient temperature change $\delta T(t)$ after the pulse but also on the steady-state temperature $T$. The determination of the steady-state temperature increase $\Delta T$ above the base temperature $T_{b}$ under the pump laser spot is therefore important in order to assess quantitatively the light excitation mechanism and efficiency. Moreover, for spatially resolved studies, e.g., pump-assisted domain wall motion, ${ }^{14}$ magnetization reversal, ${ }^{15}$ or excitation of spin waves by surface acoustic waves, ${ }^{16,17}$ knowing the lightinduced temperature radial profile $\Delta T(r)$ would be of valuable interest in order to assess the contributions of thermal and non-thermal effects.

The magnetization precession frequency has been used in the previous studies ${ }^{8,18,19}$ as a thermometer to determine the pump-induced increase of the base temperature by comparing its dependence on temperature and on pump fluence. However, this method is not usable in cases when (i) the precession period is too long to be properly measured in the experimental time window of a few ns (e.g., for temperatures close to the Curie temperature $T_{C}$ ), or (ii) when precession is absent from the transient dynamical signal. ${ }^{20,21}$ Moreover, it does not allow the determination of the radial temperature profile since the pump-induced dynamical signal is lost as soon as the pump and probe spots do not overlap. In non-magnetic materials, one can probe the temperature profile arising from heat diffusion from the thermally induced variation of reflectance across a pump laser spot. ${ }^{22,23}$ However, in such a thermoreflectance experiment, only the temperature profile is obtained, not the absolute temperature.

To determine the pump-induced temperature rise and the thermal gradient around the pump spot, we present an original method relying on the thermal dependence of the magnetic and magneto-optical effects. We use the hysteresis cycles observed through the Voigt effect ${ }^{24}$ in GaMnAs ${ }^{25}$ (also called magnetic linear dichroism/birefringence). The spatial profile of the pump-induced temperature increase is obtained by scanning the pump beam across the probe spot. The data are analyzed within the heat diffusion model from which we deduce the thermal conductivity and the contact resistance at the sample-thermostat interface.

\section{SAMPLE AND EXPERIMENTAL SETUP}

The sample is a $50 \mathrm{~nm}$ thick $(\mathrm{Ga}, \mathrm{Mn}) \mathrm{As}_{0.957} \mathrm{P}_{0.043}$ epilayer grown by molecular beam epitaxy on a $350 \mu \mathrm{m}$ (001) semi-insulating GaAs substrate. It is annealed $1 \mathrm{~h}$ at $250{ }^{\circ} \mathrm{C}$. The effective $\mathrm{Mn}$ concentration is $4 \%$. The $T_{C}$ $(85 \mathrm{~K})$ and anisotropy constants were determined from superconducting quantum interference device magnetometry and ferromagnetic resonance experiments. ${ }^{13}$ Below $60 \mathrm{~K}$, the 
magnetic anisotropy is biaxial with easy axes between the inplane $\langle 100\rangle$ and $\langle 110\rangle$ directions. The sample is glued with silver paste on the copper cold finger of an optical helium flow cryostat. The laser source is a $76 \mathrm{MHz}$ Ti:Sa laser with a pulse width of $\approx 200 \mathrm{fs}$. The beam is split into pump and probe beams modulated at $f_{\text {pump }}=50 \mathrm{kHz}$ and $f_{\text {probe }}=520 \mathrm{~Hz}$, respectively. A microscopy setup with resolution $\approx 2 \mu \mathrm{m}$ is used to monitor the spatial profile of the pump and probe spots and their respective coordinates. Their profiles are fitted with Gaussian functions with radius at $1 / e^{2} w=9 \mu \mathrm{m}$ for the probe and $w=17 \mu \mathrm{m}$ to $22 \mu \mathrm{m}$ for the pump. The incident fluence is calculated as $F=E_{p} / \pi w^{2}$ with $E_{p}$, the energy per pulse. The probe beam fluence is $F_{\text {probe }}=0.23 \mu \mathrm{J} \mathrm{cm}^{-2}$. The experiments were performed at a wavelength $\lambda=700 \mathrm{~nm}$, for significant magneto-optical signal.

\section{CORRESPONDENCE BETWEEN FLUENCE AND TEMPERATURE}

\section{A. Temperature versus fluence from hysteresis cycles}

Magneto-optical effects induce a rotation of the probe beam polarization which is detected by a balanced optical diode bridge whose signal is fed into a lock-in amplifier. The static magneto-optical signal is detected at frequency $f_{\text {probe }}$ while the field is cycled along the [1-10] axis. The even component of the hysteresis cycle that arises from the Voigt effect quadratic in the magnetization $M$ is shown in Figs. 1(a) and 1(b). The cycle shape with a continuous variation of the signal (path $(1,4))$ and switching steps $\left(2\right.$ at field $H_{u}$ and 3 at field $H_{d}$ ) is typical of an in-plane biaxial magnetic anisotropy. ${ }^{26}$ The reversal mechanism from $\mu_{0} H=-20$ to $+20 \mathrm{mT}$ is schematically depicted in Fig. 1(c). The magnetization orientations at $\mu_{0} H= \pm 20 \mathrm{mT}$ and $\mu_{0} H \approx 0$ at $T=10 \mathrm{~K}$ are calculated from the minimization of the magnetic energy. ${ }^{27}$ Any infinitesimal misalignment of the field with [1-10] will determine the direction of magnetization rotation.

Figure 1(a) shows the Voigt cycles obtained without pump beam as a function of the base temperature. Fig. 1(b) shows the same cycles obtained with overlapping probe and pump spots, at a base temperature $T_{b}=10.5 \mathrm{~K}$ as a function of the pump fluence. We checked that the cycles do not depend on the delay between the pump and the probe pulses and that increasing the probe fluence by a factor of 10 has no effect. The amplitude and switching field $H_{d}$ of the two sets of cycles in Fig. 1 evolve very similarly, decreasing with increasing temperature or fluence, thus giving strong evidence that raising the pump fluence is equivalent to raising the steady-state temperature. More specifically, we expect the amplitude of the cycle to vary like $M(T)^{2} \cos 2 \phi_{0}(T, H \approx 0)$ where $\phi_{0}$ is the equilibrium orientation of the magnetization. The switching fields $H_{u}$ and $H_{d}$ reflect the nucleation and expansion of $90^{\circ}$ domains. ${ }^{28}$ These switching fields are governed by temperature and fielddependent energy barriers $E_{2}$ and $E_{3}$, respectively. Barrier $E_{2}$ is higher than $E_{3}$ as found from the angular dependence of the magnetic energy. Switching step 2 at $H_{u}$ is therefore mainly governed by nucleation on defects (most likely located away from the laser spot) and hardly sensitive to the temperature contrary to switching step 3 at $H_{d}$. Therefore, the correspondence between fluence and temperature is extracted using the values of the cycle amplitude and down-switching field $H_{d}$ (at half amplitude) of the Voigt cycles in Fig. 1, the latter being much more sensitive owing to the $\exp \left(-E_{3} / k_{B} T\right)$ dependence of the switching probability.

Figure 2 shows the resulting steady-state temperature as a function of the pump fluence. The temperatures extracted from the cycle amplitude (full squares) and from $H_{d}$ (triangles) show similar behaviors. Two regimes are observed. The temperature rises with pump fluence with a slope of

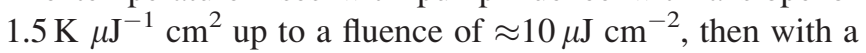

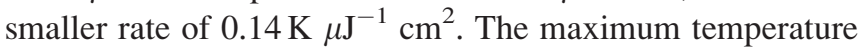
rise is of about $25 \mathrm{~K}$ for a fluence of $100 \mu \mathrm{J} \mathrm{cm}^{-2}$ at a base temperature $T_{b}=10.5 \mathrm{~K}$.

\section{B. Temperature versus fluence from precession frequency}

We now compare the temperature obtained from the hysteresis cycle to that deduced from the precession frequency. Magnetization precession induces a dynamic rotation of the probe beam polarization plane via the magneto-optical effects. ${ }^{29}$ The dynamical signal is detected in a double demodulation scheme at $f_{\text {pump }}$ and $f_{\text {probe }}$ and recorded as a function of the pump-probe delay. Oscillations originating
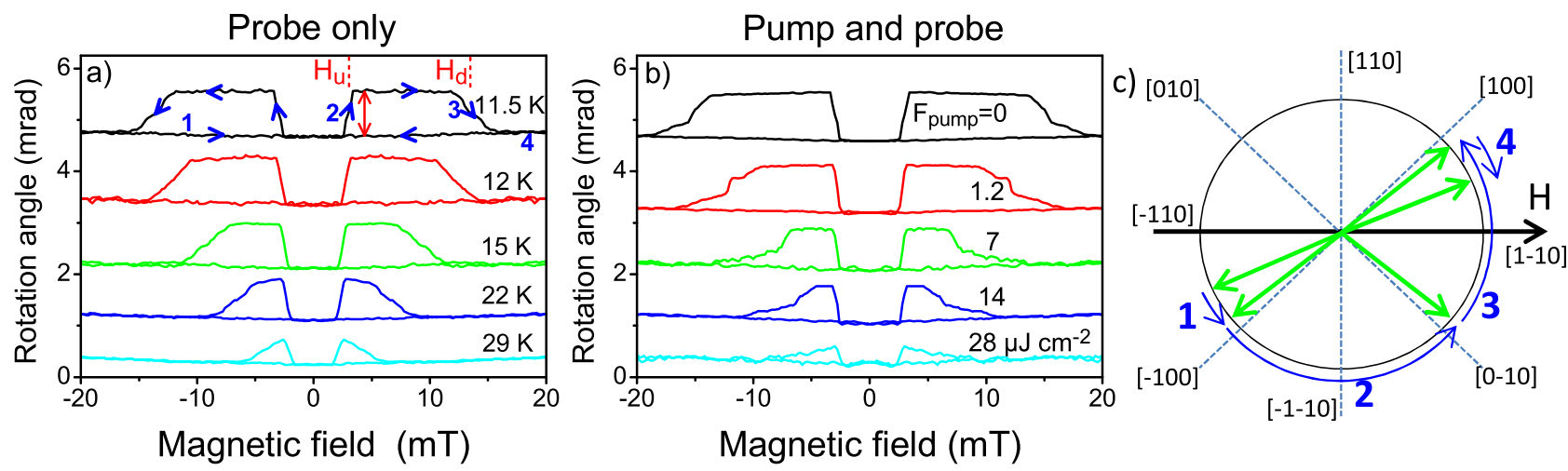

FIG. 1. Voigt hysteresis cycles: (a) for various base temperatures with probe beam only and (b) with probe and pump beam, for various pump fluences at fixed base temperature $T_{b}=10.5 \mathrm{~K}$. The pump and probe beam are linearly polarized along the [1-10] direction and the external magnetic field is applied along [1-10]. The cycles are shifted for clarity. The double-headed arrow in (a) indicates the cycle amplitude. (c) Schematics of the magnetization vector (green arrows) trajectory in an applied field along [1-10] for steps 1-4 as indicated in (a). 


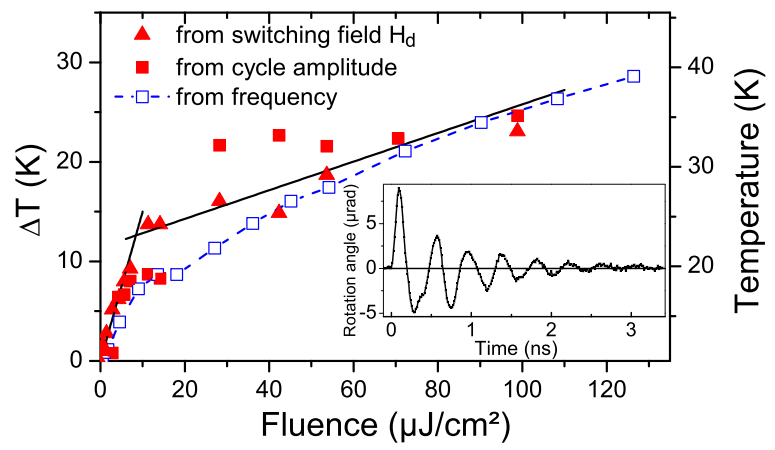

FIG. 2. Temperature increase $\Delta T$ (left scale) and temperature $T$ (right scale) versus pump fluence determined from the amplitude (full squares) and switching field $H_{d}$ (triangles) of the Voigt hysteresis cycles. The full lines represent linear fits of this ensemble of data. Empty squares: temperature from the precession frequency of the dynamical pump-probe signal (inset: typical signal at $T=10.5 \mathrm{~K}$ and $F_{\text {pump }}=2 \mu \mathrm{J} \mathrm{cm}{ }^{-2}$ ). The dashed line is a guide for the eyes.

from the excitation of two perpendicular standing spinwaves ${ }^{10}$ are observed (see inset of Fig. 2). The temperature dependence of the dynamical signal is recorded with weak pump and probe fluences $\left(F_{\text {probe }}=0.7 \mu \mathrm{J} \mathrm{cm}^{-2}, F_{\text {pump }}=2 \mu \mathrm{J}\right.$ $\mathrm{cm}^{-2}$ ). The fluence dependence of the signal is recorded at $T_{b}=10.5 \mathrm{~K}$. The temperature versus fluence dependence extracted from the fundamental frequency is shown in Fig. 2 by the open square symbols. The trend is similar to that obtained from the hysteresis cycles: an initial linear temperature rise followed by a sublinear one. The results from the frequency are consistent with those of Refs. 8, 18, and 19 both in the two-regime trend and the order of magnitude of the temperature increase versus fluence. The quantitative differences may arise from different experimental conditions, i.e., cooling by a cold finger or in the exchange gas, or from the estimation of the spot size, hence of the fluence.

\section{RADIAL THERMAL GRADIENT}

\section{A. Experimental results}

Probing the radial extent of the pump-induced temperature increase is not possible with the dynamical signal. The excited exchange-related spin waves do not propagate in the layer plane or are highly damped. The signal is lost as soon as the pump and probe cease to overlap. The Voigt hysteresis cycle, on the contrary, depends on the magnitude and equilibrium easy axes of the magnetization so that a pumpinduced modification of these quantities by a temperature change can be detected far away from the pump spot, thus allowing a spatial mapping of the temperature rise on the sample surface.

Figure 3(a) shows the temperature profile obtained from the $H_{d}$ field of the hysteresis cycles as a function of the pump and probe spot separation for a pump fluence of $17.5 \mu \mathrm{J} \mathrm{cm}^{-2}$. Results obtained from the amplitude of the hysteresis cycles are not displayed because of a much larger scattering of the values. As stated previously, this is due to the Arrhenius law for the switching probability that makes the switching field $H_{d}$ a more sensitive thermometer than the amplitude. The temperature rise at the pump spot center is
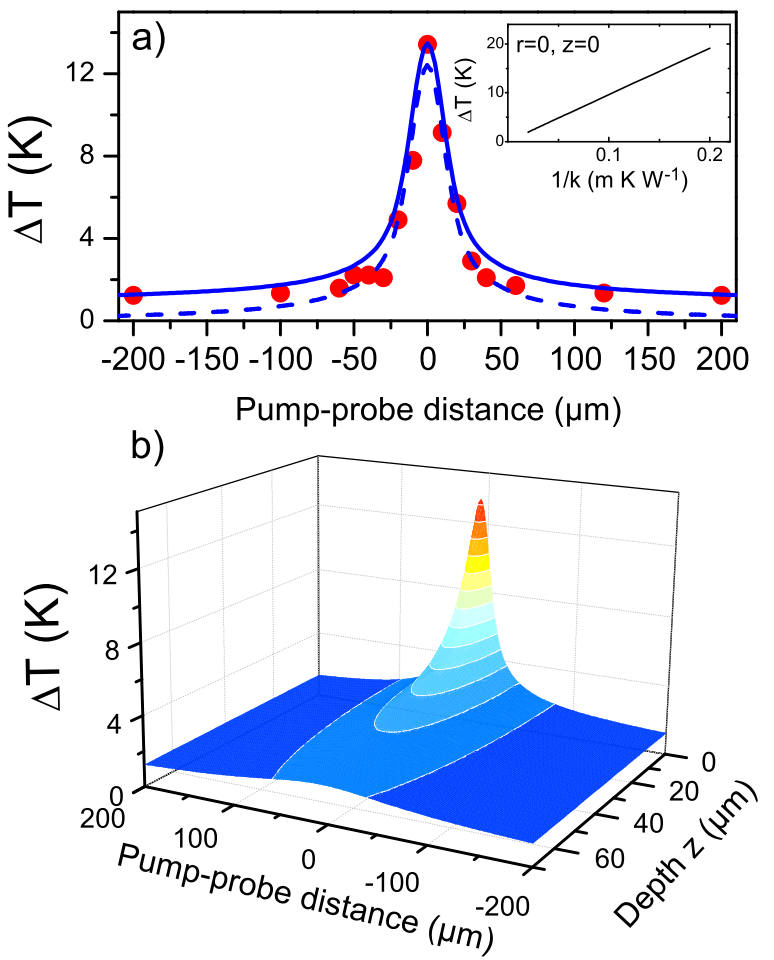

FIG. 3. (a) Radial profile of the pump-induced temperature increase at the sample surface $(z=0)$ for $F_{\text {pump }}=17.5 \mu \mathrm{J} \mathrm{cm}{ }^{-2}, w=17 \mu \mathrm{m}$ : experiment (symbols), heat diffusion model (Eq. (2)) with thermal conductivity $k=7.1 \mathrm{~W} \mathrm{~m}^{-1} \mathrm{~K}^{-1}$ and contact thermal resistance $R=0.089 \mathrm{~m}^{2} \mathrm{~K} \mathrm{~W}^{-1}$ (full line) and $R=0$ (dashed line). Parameters: $\alpha=1.4 \mu \mathrm{m}^{-1}, \Re=0.33$ (at $\lambda=700 \mathrm{~nm}$ ). Inset: calculated temperature rise at the spot center versus thermal conductivity. (b) Radial and in-depth profiles of the pump-induced temperature increase calculated from Eq. (2).

$13.5 \mathrm{~K}$ and decreases with a profile close to that of the pump. However, a temperature increase of about $1 \mathrm{~K}$ persists far from the pump center. It is even detectable $700 \mu \mathrm{m}$ away, corresponding to a change of the $H_{d}$ field by $2 \mathrm{mT}$. Note that the $H_{u}$ field hardly varies with the pump-probe separation, indicating that it corresponds to a nucleation event far from the spots as verified by longitudinal Kerr microscopy (not shown here). In the following, we show that the full temperature profile can be well reproduced within a heat diffusion model.

\section{B. Model and discussion}

Although the pump is modulated at $76 \mathrm{MHz}$ and $50 \mathrm{kHz}$, we do not need to solve the time-dependent heat diffusion equation $\partial \Delta T / \partial t-D \nabla^{2} \Delta T=p(r, z, t) / \rho C$, with $\Delta T=T-T_{b}, D$ being the diffusivity, $p$ the absorbed power per unit volume, $\rho$ the mass density, and $C$ the specific heat. Indeed, since the equation is linear, it is straightforward to show that the Fourier temperature component at $\omega$, in response to $p(t)$, is identical to the temperature in response to an input power equal to the $\omega$ component of $p(t)$. Then the continuous component of $\Delta T$, which is the measured quantity, is found as the solution of the time-independent diffusion equation in response to the timeaveraged absorbed power.

First, a two-layer sample is modeled: the epilayer with thermal conductivity $k_{1}$ and thickness $L_{1}$ and the GaAs substrate with thermal conductivity $k_{0}$ and thickness $L_{0}$. The 
following assumptions and boundary conditions are used: the energy flux from the laser transmitted at the surface $(z=0)$ is totally converted into heat flux within an infinitely thin depth, the temperature and the heat flux at the layer/substrate interface are continuous functions of the depth $z$, the sample is perfectly thermalized at the substrate-thermostat interface, i.e., $\Delta T\left(r, L_{0}+L_{1}\right)=0$. The temperature increase at the surface $(z=0)$ is thus obtained as

$$
\begin{aligned}
\Delta T(r) & =\frac{P_{0}(1-\Re)}{2 \pi} \int_{0}^{\infty} f(u) e^{-\frac{1}{8} u^{2} w^{2}} J_{0}(r u) d u \\
f(u) & =\frac{k_{1} \cosh \left(u L_{1}\right) \sinh \left(u L_{0}\right)+k_{0} \sinh \left(u L_{1}\right) \cosh \left(u L_{0}\right)}{k_{1}\left(k_{1} \sinh \left(u L_{1}\right) \sinh \left(u L_{0}\right)+k_{0} \cosh \left(u L_{1}\right) \cosh \left(u L_{0}\right)\right)},
\end{aligned}
$$

with $J_{0}$ being the Bessel function, $P_{0}$ being the average incident power, and $\Re$ being the reflectance. The fit to the experimental data with trial $k_{0}$ and $k_{1}$ values indicates that the epilayer has a lower conductivity than the substrate. This confirms a room temperature thermoreflectance estimation of the conductivity in a $50 \mathrm{~nm}$ GaMnAs layer: ten times smaller than that of the GaAs substrate. ${ }^{30}$ The $(\mathrm{Ga}, \mathrm{Mn})(\mathrm{As}, \mathrm{P})$ or GaMnAs layers, despite their metallic character (high carrier density of a few $10^{20} \mathrm{~cm}^{-3}$ ) show a poor heat conductivity, presumably because of phonon scattering by the Mn dopants and well-known defects like As antisites.

Since $\Delta T$ is mostly sensitive to $k_{0}$, much less to $k_{1}$, we can simplify the system and take into account a single layer with thickness $L=L_{0}+L_{1}$ and effective thermal conductivity $k$. To be more exhaustive, we include the light absorption depth $1 / \alpha$ and solve the heat diffusion equation $\nabla^{2} \Delta T=-p(r, z) / k \quad$ with $\quad p(r, z)=\left(2 \alpha P_{0}(1-\Re) / \pi w^{2}\right)$ $\exp \left(-2 r^{2} / w^{2}\right) \exp (-\alpha z)$. With a perfectly thermalized substrate $(\Delta T(r, z=L)=0)$, the resulting $\Delta T$ agrees quite well with the data close to the epicenter (dashed curve in Fig. 3(a)) but fails to reproduce the temperature rise persisting far from it. For this, we have to take into account a contact thermal resistance $R$ at the substrate-thermostat interface introduced as $\left.R k \frac{\partial \Delta T}{\partial z}\right|_{z=L}=\Delta T\left(\lambda, L^{+}\right)-\Delta T\left(\lambda, L^{-}\right)$ with $\Delta T\left(\lambda, L^{+}\right)=0$. The temperature profile is then obtained as

$$
\begin{aligned}
\Delta T(r, z)= & \frac{P_{0}(1-\Re)}{2 \pi} \int_{0}^{\infty} g(u, z) e^{-\frac{1}{8} u^{2} w^{2}} J_{0}(r u) d u \\
g(u, z)= & \frac{\alpha}{k\left(u^{2}-\alpha^{2}\right)} \\
& \times\left(\frac{\cosh (u z)\left(\alpha e^{-L u}(1-k R u)+u e^{-\alpha L}(\alpha k R-1)\right)}{k R u \sinh (L u)+\cosh (L u)}\right. \\
& \left.+u e^{-\alpha z}-\alpha e^{-u z}\right) .
\end{aligned}
$$

The temperature profile calculated from Eq. (2) at $z=0$ is now shown in Fig. 3(a) by a full line. From the best fit at the spot center $(r=0)$ and wings $(r=200 \mu \mathrm{m})$, we find a thermal conductivity $k=7.1 \mathrm{~W} \mathrm{~m}^{-1} \mathrm{~K}^{-1}$ and a contact resistance $R=0.089 \mathrm{~m}^{2} \mathrm{~K} \mathrm{~W}^{-1} . k$ is mostly determined by the temperature at the spot center $(r=0)$ and $R$ by the temperature at large $r$. The fit is quite sensitive to $k$ since $\Delta T$ at the spot center varies as $1 / k$ (inset of Fig. 3(a)). In the depth, the temperature rise is important only in a few tens of micrometers close to the surface as shown in Fig. 3(b).

The $k$ value is low but reasonable compared to data on doped GaAs at low temperature (below $50 \mathrm{~W} \mathrm{~m}^{-1} \mathrm{~K}^{-1}$ at $10 \mathrm{~K}),{ }^{31}$ where phonon scattering by impurities is important. The thermal conductivity increases rapidly with temperature. In a similar system, a thermal conductivity of $140 \mathrm{~W} \mathrm{~m}^{-1}$ $\mathrm{K}^{-1}$ was obtained from transport measurements in tracks at $100 \mathrm{~K}^{32}$

Let us note that, within the heat diffusion model, $\Delta T$ is proportional to the pump power, hence to the fluence, and inversely proportional to the thermal conductivity. The change from a linear regime at low pump fluence to a sublinear one at high pump fluence observed experimentally hence means that the assumption of a thermal conductivity depending only on the base temperature is most likely not valid anymore when the thermal conductivity varies strongly with the temperature and $\Delta T$ is not small with respect to $T_{b}$. However, solving the diffusion equation with $k$ depending on the local temperature $T(r, z)$ is beyond the scope of this paper. The sublinear increase of temperature with pump fluence is nevertheless in qualitative agreement with an increase of the thermal conductivity with temperature at low temperature. $^{31}$

\section{CONCLUSION}

In conclusion, using the temperature-dependence of the magneto-optical properties, more precisely the high sensitivity of the switching field to temperature, we have developed a novel method based on the Voigt hysteresis cycles to determine the pump-induced steady-state temperature increase in pump-probe experiments on magnetic layers and to spatially resolve it. This method, which gives us direct access to the magnon temperature, could be readily extended to any magnetic materials showing a hysteresis cycle whether it arises from magnetic linear birefringence/dichroism, polar, or longitudinal Kerr effect. Patterning the layer in small magnetic structures would improve the method by ensuring that all the switching fields are determined by local properties. Experiments on a $(\mathrm{Ga}, \mathrm{Mn})(\mathrm{As}, \mathrm{P})$ ferromagnetic semiconductor layer show that the temperature increase closely follows the pump profile but a small temperature increase can still be detected hundreds of micrometers away from the pump spot, which can be taken into account by a substrate-thermostat contact resistance. We have determined the low temperature thermal conductivity $k=7.1 \mathrm{~W} \mathrm{~m} \mathrm{~m}^{-1}$. The diffusivity $D$ can then be obtained provided the specific heat is known, which allows solving the time-dependent diffusion equation. This opens the way to a quantitative modeling of the space and time-resolved magnetization dynamics driven by light-induced thermal transient effects. Last but not least, for materials where the thermal conductivities and the optical index are known, our work provides a simple method to calculate the temperature gradient induced by a laser spot. 


\section{ACKNOWLEDGMENTS}

We are grateful to M. Bernard, F. Breton, and F. Margaillan (INSP) for technical assistance. This work has been supported by UPMC (Emergence 2012), Region Ile-deFrance (DIM Nano-K MURAS2012), and Agence Nationale de la Recherche (Project No. ANR13-JS04-0001-01).

${ }^{1}$ A. Hoffmann and S. D. Bader, Phys. Rev. Appl. 4, 047001 (2015).

${ }^{2}$ A. Khitun, M. Bao, and K. L. Wang, J. Phys. D: Appl. Phys. 43, 264005 (2010).

${ }^{3}$ J. Hohlfeld, T. Gerrits, M. Bilderbeek, T. Rasing, H. Awano, and N. Ohta, Phys. Rev. B 65, 012413 (2001).

${ }^{4}$ A. H. M. Reid, G. V. Astakhov, A. V. Kimel, G. M. Schott, W. Ossau, K. Brunner, A. Kirilyuk, L. W. Molenkamp, and T. Rasing, Appl. Phys. Lett. 97, 232503 (2010).

${ }^{5}$ M. van Kampen, C. Jozsa, J. Kohlhepp, P. LeClair, L. Lagae, W. de Jonge, and B. Koopmans, Phys. Rev. Lett. 88, 227201 (2002).

${ }^{6}$ D. Wang, Y. Ren, X. Liu, J. Furdyna, M. Grimsditch, and R. Merlin, Phys. Rev. B 75, 233308 (2007).

${ }^{7}$ Y. Hashimoto, S. Kobayashi, and H. Munekata, Phys. Rev. Lett. 100, 067202 (2008)

${ }^{8}$ J. Qi, Y. Xu, A. Steigerwald, X. Liu, J. Furdyna, I. Perakis, and N. Tolk, Phys. Rev. B 79, 085304 (2009).

${ }^{9}$ P. Němec, E. Rozkotová, N. Tesařová, F. Trojánek, E. De Ranieri, K. Olejník, J. Zemen, V. Novák, M. Cukr, P. Malý, and T. Jungwirth, Nat. Phys. 8, 411 (2012)

${ }^{10}$ S. Shihab, H. Riahi, L. Thevenard, H. J. von Bardeleben, A. Lemaitre, and C. Gourdon, Appl. Phys. Lett. 106, 142408 (2015).

${ }^{11}$ A. Kirilyuk, A. Kimel, and T. Rasing, Rev. Mod. Phys. 82, 2731 (2010).

${ }^{12}$ Y. Liu, L. R. Shelford, V. V. Kruglyak, R. J. Hicken, Y. Sakuraba, M. Oogane, and Y. Ando, Phys. Rev. B 81, 094402 (2010).

${ }^{13}$ M. Cubukcu, H. J. von Bardeleben, K. Khazen, J. L. Cantin, O. Mauguin, L. Largeau, and A. Lemaître, Phys. Rev. B 81, 041202 (2010).

${ }^{14}$ A. J. Ramsay, P. E. Roy, J. A. Haigh, R. M. Otxoa, A. C. Irvine, T. Janda, R. P. Campion, B. L. Gallagher, and J. Wunderlich, Phys. Rev. Lett. 114, 067202 (2015).
${ }^{15}$ G. Astakhov, H. Hoffmann, V. Korenev, T. Kiessling, J. Schwittek, G. Schott, C. Gould, W. Ossau, K. Brunner, and L. Molenkamp, Phys. Rev. Lett. 102, 187401 (2009).

${ }^{16}$ Y. Au, M. Dvornik, T. Davison, E. Ahmad, P. S. Keatley, A. Vansteenkiste, B. Van Waeyenberge, and V. V. Kruglyak, Phys. Rev. Lett. 110, 097201 (2013).

${ }^{17}$ N. Ogawa, W. Koshibae, A. J. Beekman, N. Nagaosa, M. Kubota, M. Kawasaki, and Y. Tokura, Proc. Natl. Acad. Sci. U.S.A. 112, 8977 (2015).

${ }^{18} \mathrm{~N}$. Tesařová, P. Němec, E. Rozkotová, J. Zemen, T. Janda, D. Butkovičová, F. Trojánek, K. Olejník, V. Novák, P. Malý, and T. Jungwirth, Nat. Photonics 7, 492 (2013).

${ }^{19}$ H. Li and X.-H. Zhang, Chin. Phys. Lett. 32, 067501 (2015).

${ }^{20}$ Y. Mitsumori, A. Oiwa, T. Supinski, H. Maruki, Y. Kashimura, F. Minami, and H. Munekata, Phys. Rev. B 69, 033203 (2004).

${ }^{21}$ K. C. Hall, J. P. Zahn, A. Gamouras, S. March, J. L. Robb, X. Liu, and J. K. Furdyna, Appl. Phys. Lett. 93, 032504 (2008).

${ }^{22}$ A. Rosencwaig, J. Opsal, W. L. Smith, and D. L. Willenborg, Appl. Phys. Lett. 46, 1013 (1985).

${ }^{23}$ C. Fretigny, J.-Y. Duquesne, D. Fournier, and F. Xu, J. Appl. Phys. 111, 084313 (2012).

${ }^{24}$ A. K. Zvezdin and V. A. Kotov, Modern Magnetooptics and Magnetooptical Materials (CRC Press, New York, 1997).

${ }^{25}$ A. V. Kimel, G. V. Astakhov, A. Kirilyuk, G. M. Schott, G. Karczewski, W. Ossau, G. Schmidt, L. W. Molenkamp, and T. Rasing, Phys. Rev. Lett. 94, 227203 (2005).

${ }^{26}$ G. P. Moore, J. Ferré, A. Mougin, M. Moreno, and L. Däweritz, J. Appl. Phys. 94, 4530 (2003).

${ }^{27}$ M. Cubukcu, H. J. von Bardeleben, J. L. Cantin, and A. Lemaître, Appl. Phys. Lett. 96, 102502 (2010).

${ }^{28}$ U. Welp, V. K. Vlasko-Vlasov, and T. Wojtowicz, Phys. Rev. Lett. 90, 167206 (2003).

${ }^{29}$ N. Tesařová, P. Nêmec, E. Rozkotová, J. Šubrt, H. Reichlová, D. Butkoviĉová, F. Trojánek, P. Malý, V. Novák, and T. Jungwirth, Appl. Phys. Lett. 100, 102403 (2012).

${ }^{30}$ D. Fournier, private communication (2016).

${ }^{31}$ R. O. Carlson, G. A. Slack, and S. J. Silverman, J. Appl. Phys. 36, 505 (1965).

${ }^{32}$ J. Curiale, A. Lemaître, G. Faini, and V. Jeudy, Appl. Phys. Lett. 97, 243505 (2010). 